\title{
HVDC System for a Data Center Equipped with SiC Power Devices
}

\author{
Tamotsu Ninomiya ${ }^{1}$, Akiyoshi Fukui ${ }^{2}$, Masato Mino ${ }^{2}$, Mikio Yamasaki ${ }^{3}$, \\ Yasunori Tanaka ${ }^{4}$, and Hiromichi Ohashi ${ }^{4}$ \\ ${ }^{1}$ Nagasaki University, Nagasaki, Japan \\ ${ }^{2}$ NTT Facilities Inc., Tokyo, Japan \\ ${ }^{3}$ NTT Facilities Research Institute Inc., Tokyo, Japan \\ ${ }^{4}$ National Institute of Advanced Industrial Science and Technology, Tsukuba, Japan
}

\begin{abstract}
SiC power devices have attracted attention as the next-generation semiconductor devices that surpass silicon devices. The SiC has some superior physical properties when compared with the conventional silicon, and is expected to operate with lower on-resistance and under higher temperature. Furthermore the SiC device has higher thermal conductivity and then has the prominent feature of heat dissipation. Among many expected applications of $\mathrm{SiC}$, this paper presents the application to the power supply system for the information and communication system such as a data center. Firstly, the development of a high power-density technology for a 5-kW isolated DC-DC converter using a hybrid-pair of Si-MOSFET and SiC-SBD is reported. Secondly, a new type DC circuit breaker using SiC-SIT is introduced.
\end{abstract}

\section{INTRODUCTION}

In recent years, the amount of worldwide internet traffic has been extraordinary increased because of the high performance of personal computers and various portable terminals, and the widespread use of optical communication networks. Correspondingly the volume of communication processing in data centers has been increased, and then the scale of data centers has been expanded. At the same time, the power consumption in data centers has also been increased, and the energy saving has been required. As a result, the efficiency improvement of power supply systems for ICT equipment has been also demanded [1].

In the conventional data center, the power supply system consists of alternating current (AC) systems, and the AC power of $100-200 \mathrm{~V}$ is distributed to ICT equipment as shown in Fig. 1(a), where four stages of power conversion are used and the efficiency of the power supply system is low. On the other hand, the direct current (DC) is utilized for decrease in the number of power conversion stages in the power distribution, and presently $48 \mathrm{VDC}$ is commonly used. However, when the power required in ICT equipment is increased and the distribution current becomes large, a lot of power distribution loss occurs.

Recently, a high-voltage DC (HVDC) power supply system shown in Fig.1(b) has been attracting attention for the many advantages such as fewer conversion stages than AC supplies and finer power distribution cables than the 48-V DC system. Research on a 400V HVDC power supply system has been reported [2,3]. This HVDC power supply system has some prominent features of reducing power-conversion steps, reducing distribution current by using higher voltage, and achieving a smaller and more efficient system, which result in a so-called "high power-density" system. On the other hand, the higher voltage of $400 \mathrm{~V}$ means that the withstand voltage above $1000 \mathrm{~V}$ is needed for semiconductor devices and other components used in this power supply system, considering the surge-voltage occurrence. From this viewpoint, the application of the recently developed $\mathrm{SiC}$ diodes is attractive.

Another technical difficulty for the DC power distribution system is "circuit breaking" for protecting a total system when a trouble of over-current or short-circuit occurs in one of many load branches. The conventional fuses and mechanical circuit breakers have some difficulties of slow breaking speed, maintenance for replacing, and inability of future multifunction operation. A possible solution to these difficulties is the application of "semiconductor circuit breakers" [2]. The first-version prototype DC circuit breakers were fabricated by using the conventional silicon devices, but the size including surge snubbers was much bigger [3]. Therefore, attention has turned to the application of silicon-carbide static-inductiontransistors (SiC-SIT) [4] which has the prominent features of low on-resistance and high withstand voltage and current.

This paper presents the development of a high power-density technology with a hybrid pair of Si-MOSFET and SiC-SBD for a $5 \mathrm{~kW}$ isolated DC-DC converter utilized at the input stage of a HVDC power supply system, and a new type semiconductor circuit breaker using SiC-SIT.

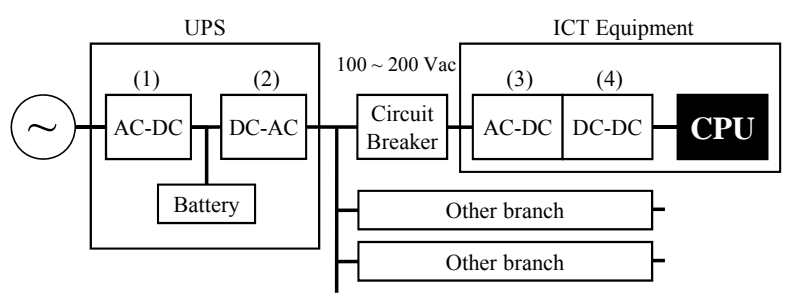

Fig.1(a) Conventional AC Power Supply System.

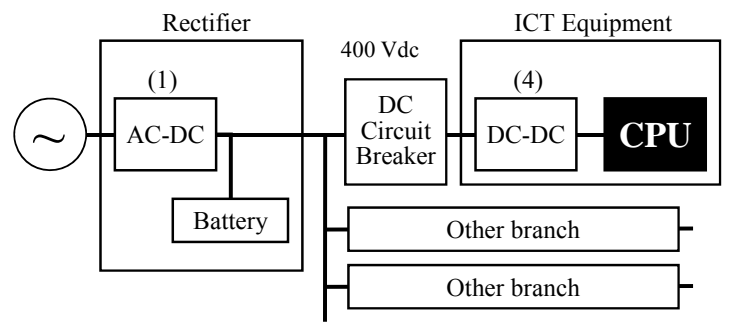

Fig.1(b) HVDC Power Supply System. 


\section{DC-DC CONVERTER UNIT WITH HIGH POWER DENSITY}

\section{A. SiC application to power converter}

Power consumption of servers, routers, and other data processing equipment has been increased with improvement of the processing capabilities of such equipment, and has reached up to an average power per floor space of $500 \mathrm{~W} / \mathrm{m}^{2}$. Accordingly, a building which has a floor area of $1000 \mathrm{~m}^{2}$, for example, would consume about $550 \mathrm{kVA}$, taking power used for ventilation fans and lighting, etc., into account. The above power supply for such data processing equipment usually utilizes the rectifiers, which convert the input of three-phase 200 VAC to the output of 48 VDC. In this case, the power for one system (i.e. one rack) is generally set to be $100 \mathrm{~kW}$. The recent development has increased the conversion frequency of these rectifiers up to several tens of kilohertz for a $400 \mathrm{VDC}$ power supply system, and the power density has been increased from $0.2 \mathrm{~W} / \mathrm{cm}^{3}$ to $0.5 \mathrm{~W} / \mathrm{cm}^{3}$ [5].

The application of $\mathrm{SiC}$ devices is expected to increase the power conversion efficiency and reduce the size of these rectifiers, and then to increase the power density. For example, if the power density of these rectifiers is increased up to 5 $\mathrm{W} / \mathrm{cm}^{3}$, the present power level can be increased by a factor of 10 , and the expected power level will be $1000 \mathrm{~kW}$ per rack. In this case, the power consumption of $500 \mathrm{~kW}$ at $1000 \mathrm{~m}^{2}$ above-mentioned can be supplied by half of a single rack.

\section{B. Configuration of the isolated DC-DC converter}

In a power supply system for ordinary communication equipment, the input-stage rectifier comprises two stages; one is a power-factor-correction (PFC) converter which suppresses the power-line harmonics current generated by diode rectification, and the other is an isolated DC-DC converter which achieves the DC isolation for safety and the output-voltage stabilization, as shown in Fig. 2(a). When this isolated DC-DC converter is required to be a high-power converter, the full-bridge type configuration shown in Fig.2(b) is selected as a typical topology. It is because the magnetic flux amplitude of the transformer core is decreased by producing a symmetrical variation, and the average current through a transistor switch each is decreased by half-cycle operation. In this full-bridge isolated DC-DC converter, as shown by the gate-voltage waveforms in Fig. 3, the switch pairs Q1-Q2 and Q3-Q4 respectively operate on and off reversely with the dead times and then the zero-voltage-switching (ZVS) operation of these switches is achieved by the transformer magnetizing current. Furthermore, the pulse width of the transformer voltage can be controlled by adjusting the phase difference $\phi$ between the turnoff times of Q1 and Q4 and the phase difference $\phi$ between the turnoff times of Q2 and Q3, and then the output voltage can be controlled.

On the other hand, a large surge voltage is generated in this DC-DC converter by transformer leak inductances Lr1 and $\mathrm{Lr} 2$ and the diode recovery characteristics at the turnoff time of secondary-side diodes. The surge may exceed the input voltage by a factor of five, and so for a $400 \mathrm{~V}$ high-voltage input, diodes with a high withstand voltage of over $2000 \mathrm{~V}$ are needed, or big surge-snubber circuits must be added. However, either case induces an obstacle to higher power density of the converter. To solve this surge problem, the insertion of a relatively large capacitor $\mathrm{Cs}$ in front of the output filter Lo and Co has been proposed, and a prominent surge-absorbing effect has been confirmed [6].

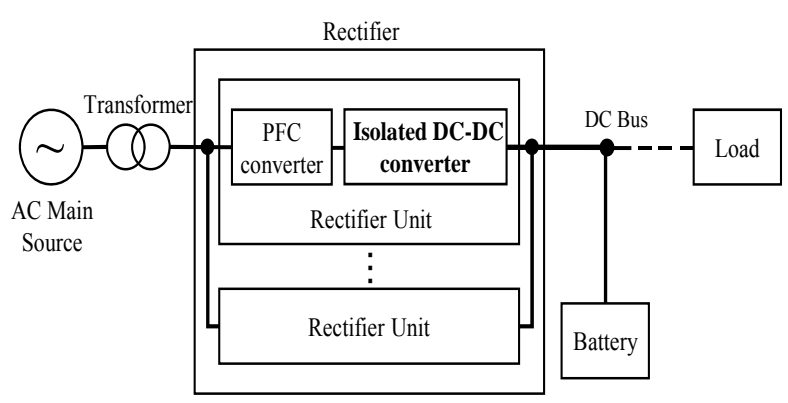

Fig.2(a) Rectifier configuration.

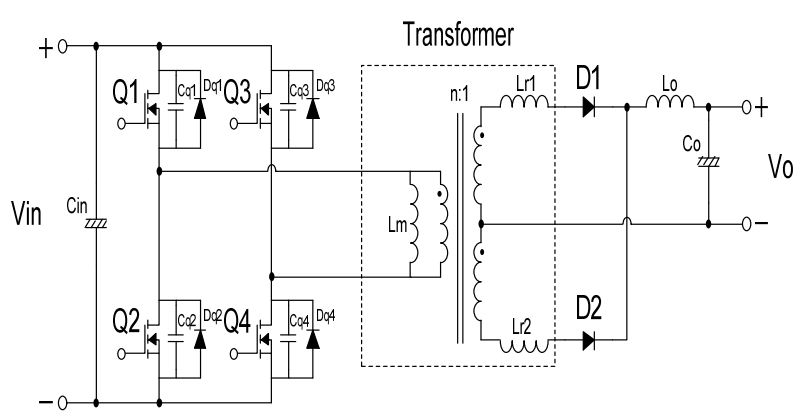

Fig.2(b) Full-bridge type isolated DC-DC converter.

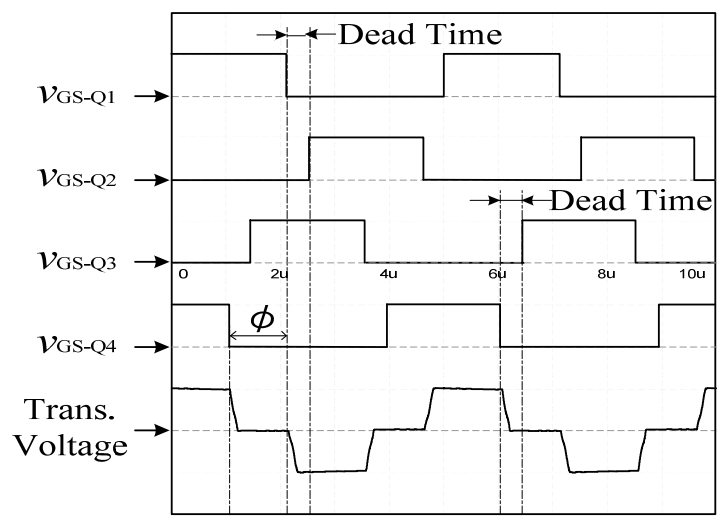

Fig.3. Driving voltage waveforms of switch and transformer.

\section{Prototype high power-density DC-DC converter}

On the basis of the research described above, a prototype of the high-power isolated DC-DC converter with the input of $400 \mathrm{~V}$ and the output of $400 \mathrm{~V} / 12.5 \mathrm{~A}(5 \mathrm{~kW})$ was fabricated [6]. The component specifications for the semiconductor devices, magnetic devices, and other components are listed in Table 1. This prototype was intended to achieve a power density of 10 $\mathrm{W} / \mathrm{cm}^{3}$, an order of magnitude much higher than the conventional one. The experimental unit shown in Fig. 4 has 
the dimensions of $7.8 \times 6 \times 10.7 \mathrm{~cm}\left(500 \mathrm{~cm}^{3}\right)$ and achieved an efficiency of $95 \%$ for an output of $4.3 \mathrm{~kW}$. The calculated volume ratios for various components are illustrated in Fig. 5. In the near future, a power density of $20 \mathrm{~W} / \mathrm{cm}^{3}$ can be expected from further reducing power loss, removing the heat sink and fan, and more compact mounting of the components.

Table 1. Circuit components of prototype DC-DC converter.

\begin{tabular}{|c|c|}
\hline CoolMOS & $900 \mathrm{~V} / 36 \mathrm{~A}$ \\
\hline SiC-SBD & $1200 \mathrm{~V} / 2 * 15 \mathrm{~A}$ \\
\hline Magnetic Core & $\begin{array}{c}\text { TDK PC 95 } \\
\text { (PQ40/40) }\end{array}$ \\
\hline Winding turns ratio & $15: 18$ \\
\hline Winding type & Foil Copper \\
\hline $\begin{array}{c}\text { Leakage inductance } \\
\text { in secondary side }\end{array}$ & $2.5 \mu \mathrm{H}$ \\
\hline Filter Inductor (Lo) & $132 \mu \mathrm{H}$ \\
\hline
\end{tabular}

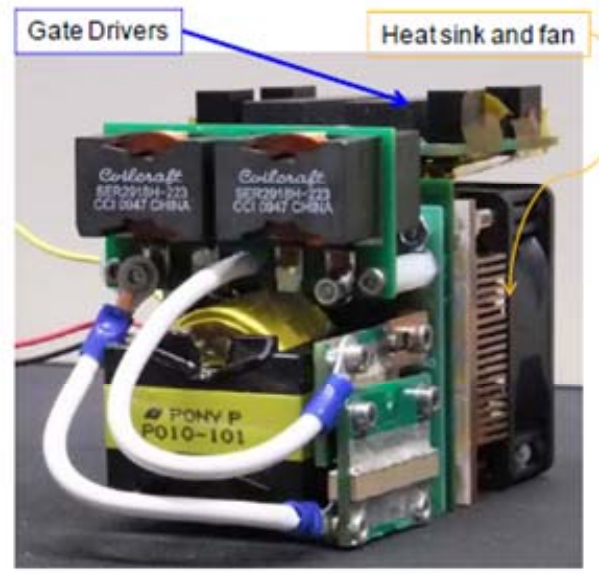

Fig.4. External view of prototype DC-DC converter.

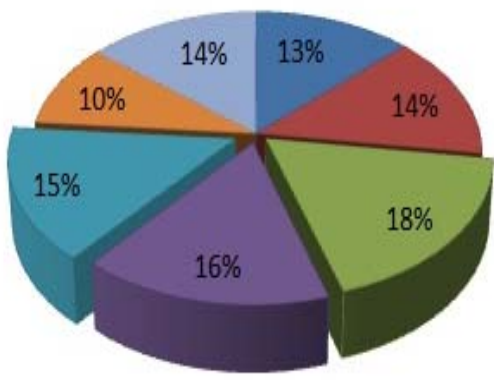

- Power Module

- Heat Sink

DFan

口output Filter

Transformer

口 Gate Driver

Vacant area

Fig.5. Volume ratio of prototype DC-DC converter.

\section{SiC-SIT DC CIRCUIT BREAKERS}

\section{A. Characteristics necessary for DC circuit breakers}

In the HVDC power supply system, the essential operation of a DC circuit breaker is to interrupt the over-current which occurs in case of troubles such as a short-circuited power line and so forth. At the time of this current interruption, a high-voltage surge arises across the DC circuit breaker due to line inductances associated with the power cable. When the conventional semiconductor device is utilized as the DC circuit breaker, a big snubber circuit is needed to protect the device and the system from high-voltage surge, and it results in an obstacle to the system downsizing. On the other hand, for the normal operation, the DC circuit breaker has to be low resistance to suppress power dissipation during its conduction. In case of the conventional semiconductor devices, a parallel connection of many devices is a possible solution for the decrease in conduction power loss, but it also results in an obstacle to the system downsizing.

In order to solve both the demands for the normal operation and the current interruption mentioned above, a new semiconductor circuit breaker utilizing a "Silicon-Carbide Static-Induction-Transistor (SiC- SIT)" has been developed.

\section{B. DC circuit breaker using "Punch-through phenomenon"}

The SiC-SIT has a low on-resistance during its conduction, and high-withstand voltage, current, and power dissipation. Then these features are suited for usage in the DC circuit breakers. Furthermore, in order to increase the currentinterruption ability, a noble control method of the gate-voltage waveform has been developed [7].

The conventional semiconductor power devices operate in on and off states on a completely binary gate voltage, and a big voltage surge occurs at its turnoff time, and a large surgesnubber circuit is needed for protecting this device. In case of SiC-SIT device driven by a binary gate-voltage waveform, a so called "punch-through mode" occurs at the turnoff time. Then a high voltage also arises across the drain-source terminals and the drain current decreases linearly, and afterward a high-frequency voltage oscillation across the drain-source terminals as shown in Fig.6(a), where waveforms are illustrated, from top to bottom, for the drain-source voltage, for the drain current, and for the applied gate voltage. In this example, the gate voltage is $0 \mathrm{~V}$ for on-state and $-20 \mathrm{~V}$ for off-state. The surge voltage across the drain-source terminals reaches up to $1140 \mathrm{~V}$, and if the power consumption during the current interruption exceeds the limit, the SiC-SIT breaks down. Furthermore the additional high-frequency ringing may cause the EMI problems.

Next, another example of the waveforms for the current interruption is shown in Fig.6 (b), where a new gate-voltage pattern is applied, i.e. the gate-voltage is varied linearly from $-7 \mathrm{~V}$ to $-9 \mathrm{~V}$ at the time of interruption as shown by the bottom waveform. As a result, the surge voltage across the drain-source terminals is suppressed at $640 \mathrm{~V}$. This value of the surge voltage is determined by the punch-through phenomenon of the SiC-SIT, so the surge voltage at the time of interruption itself can be controlled directly by the gate-voltage waveform. Furthermore, the waveforms in Fig.6 (b) illustrate that no oscillation occurs at the drain-source voltage and the drain current after the current interruption. It is because the drain-source voltage is controlled by the special gate-voltage pattern so that the drain-source voltage can reach zero smoothly when the drain current reaches zero. 


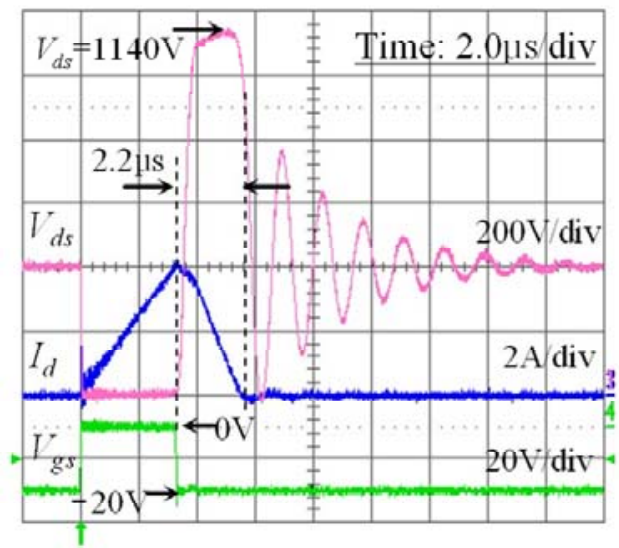

Fig.6(a) Experimental waveforms associated with SiC-SIT controlled by binary gate-voltage waveform.

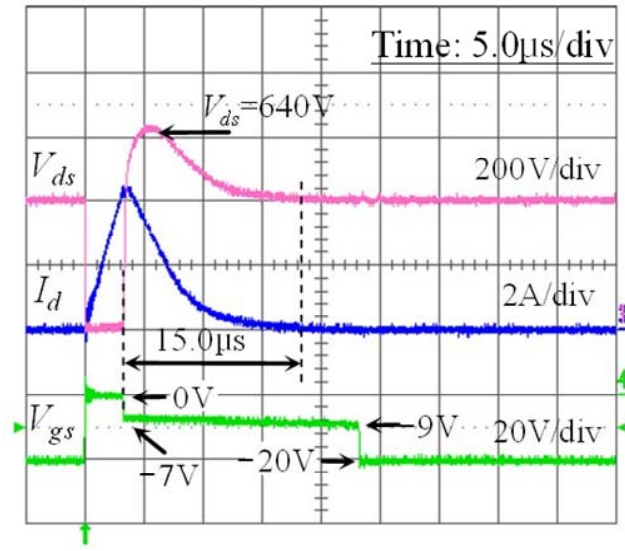

Fig.6(b) Experimental waveforms associated with SiC-SIT controlled by a new gate-voltage pattern.

\section{Practical-scale circuit breaker}

On the basis of the above-mentioned fundamental ideas and their experimental confirmation, a prototype of the practical-scale circuit breaker was fabricated and its testing was performed. This prototype has a rated voltage of $400 \mathrm{~V}$ and a rated current of $12.5 \mathrm{~A}$, which correspond to the power capacity of the power supply system in a data center. The SiC-SIT device was fabricated and mounted in TO-220 package in the laboratory. Then two SiC-SIT devices were connected in parallel and were used in this equipment.

A photograph of the prototype SiC-SIT DC circuit breaker is shown in Fig.7. The front panel holds a switch for remote control operation and LEDs to indicate the operating status, and the connecting terminals of the circuit breakers are mounted on the back side. A photograph of the circuit breakers installed in a communication system rack is shown in Fig.8. One horizontal space of the 19-inch rack can accommodate five units.

A block diagram for control panel on a personal computer is shown in Fig.9, where multiple SiC-SIT DC circuit breakers can be controlled by software via a computer network. There is continuous communication with each SiC-SIT DC circuit breaker via the network for remote control of the current interruption, and furthermore this communication network is

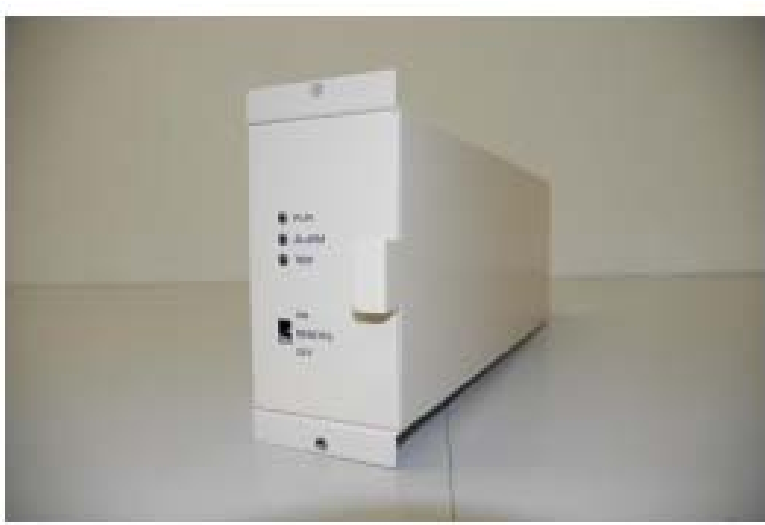

Fig.7. External view of a prototype SiC-SIT circuit breaker.

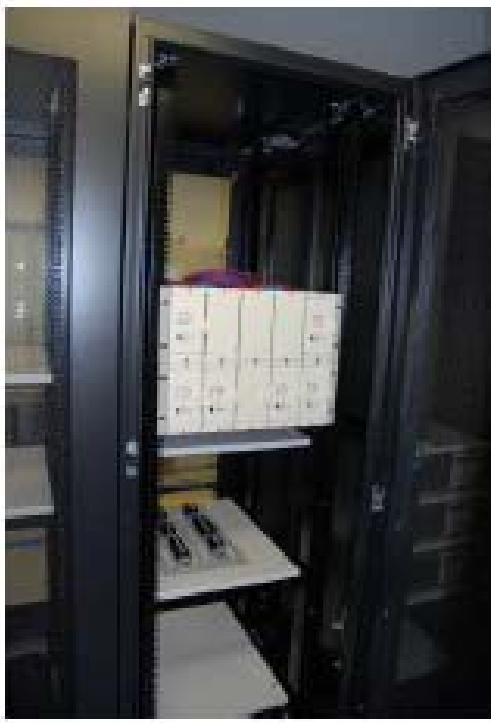

Fig.8. External view of multiple SiC-SIT circuit breakers installed in a communication system rack.

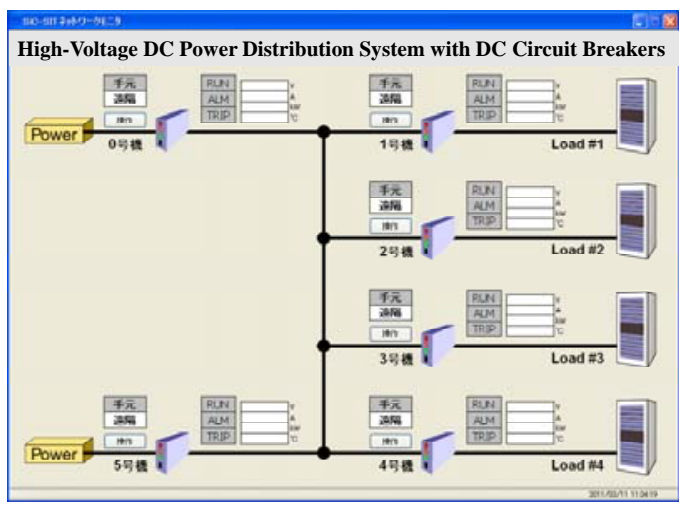

Fig.9. Block diagram for control panel on a PC, where multiple SiC-SIT circuit breakers are controlled for the current interruption.

also utilized for monitoring the voltage, current, and power status of each branch system. An actual experimental result of short-circuit current interruption by one of these circuit breakers is presented in Fig.10. In this example, a big short-circuit current of 86 A was successfully interrupted, confirming that the expected current-interruption capability was achieved. 


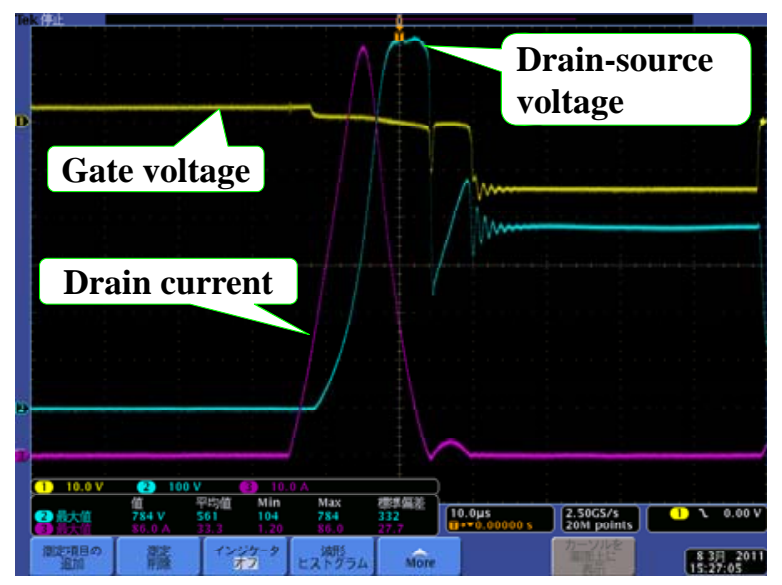

Fig.10. Test result of current interruption.

\section{FUTURE DEVELOPMENT OF POWER DISTRIBUTION SYSTEMS}

It is clarified from the above discussion that the use of $\mathrm{SiC}$ devices reduces the converter size dramatically. Then after both the safety and the certainty of current interruption by SiC-SIT DC circuit breakers are confirmed, the functionality and the intelligence of the power distribution systems for communication systems would be much advanced when compared with the present system which needs a careful operation for turning the power path on or off.

When the semiconductor circuit breakers are equipped with both the functions of communication and execution for current interruption, they would be utilized as remote switches. This means that the circuit breakers would be possible to turn the power on or off for purposes other than accidental current interruption. Such advanced functions for the converter and semiconductor circuit breaker will have a good effect on the future power distribution systems. For example, take a simple case where two power distribution systems with tree configurations of circuit breakers are connected as shown in Fig.11. Here, consider the connection of two power systems at the branching point between the top-level circuit breaker and the mid-level circuit breaker. If these semiconductor circuit breakers can be used as remote switches, the two power systems can provide mutual support according to situations such as the operational status of the load equipment or rectifier maintenance, etc. This kind of operation would be a revolutionary forward step in the power system reliability. The parallel redundant connection can be constructed by a remote control, and then the power system reliability can be changed dynamically. Furthermore, only one storage battery can be installed for the two power systems, and it will result in the efficiency increase and the cost reduction of a storage battery. These issues should be verified in future work.

Furthermore, the implementation of the advanced power system mentioned above requires a logical network which checks the state of the entire power system and decides the next action. Consequently, a new power distribution network

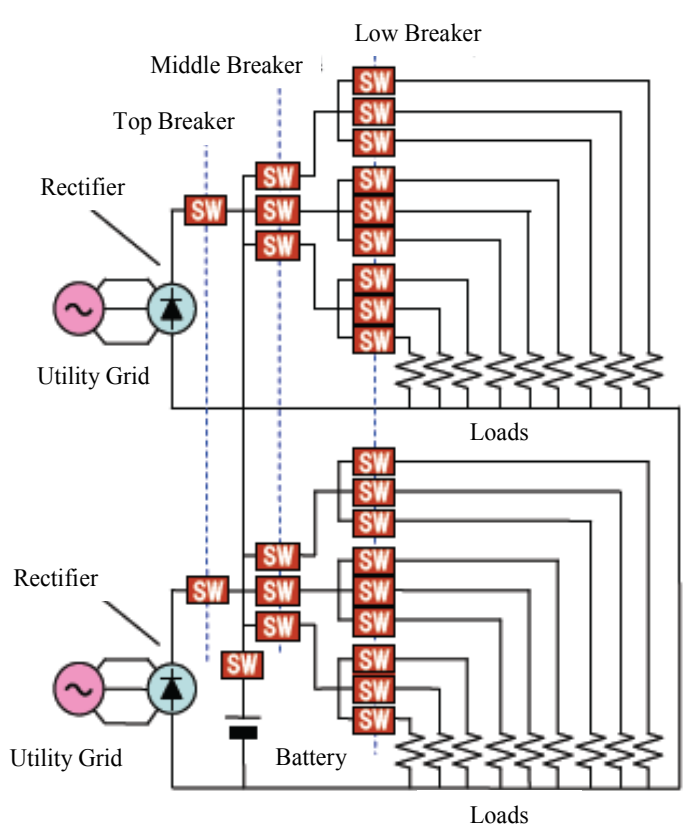

Fig.11. An example of converter connection topology in a power distribution system.

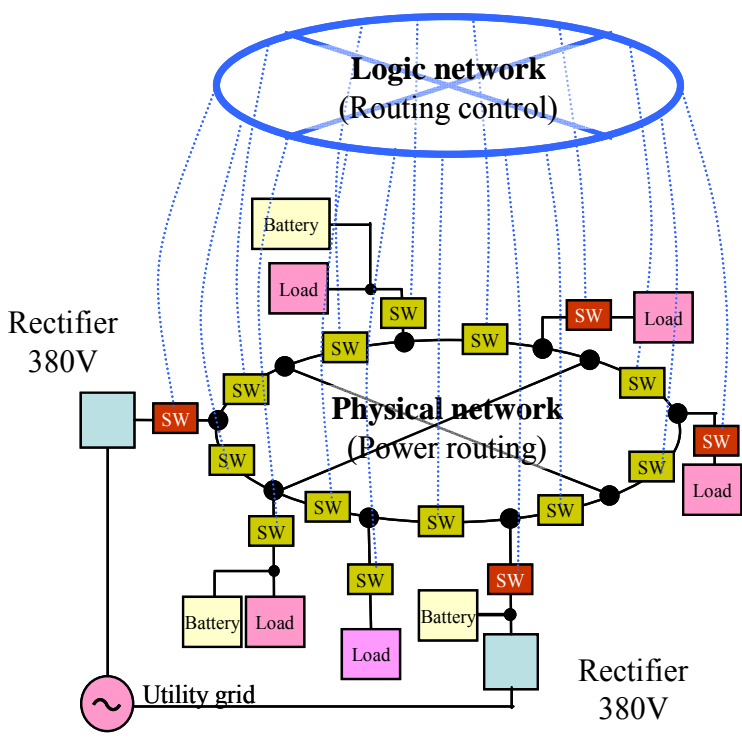

Fig.12. An example of next-generation "Power Routing System."

where the power flow is controlled by multiple bilateral circuit breakers is imagined, and its schematic diagram is shown in Fig.12. The power system has a loop and mesh topology, and information of voltage and current at multiple points are transferred to the logical network. According to the system demands, e.g. the total system efficiency, the logical network determines the optimum power flow, and controls multiple circuit breakers remotely. This scheme is named "Power Routing." 


\section{CONCLUSIONS}

The development of a high power-density converter with a hybrid pair of Si-MOSFET and SiC-SBD, and a DC circuit breaker using SiC-SIT and their application to the HVDC power distribution system have been discussed and are summarized as follows:

(1) The target for a high power-density converter was a major improvement of power density up to $10 \mathrm{~W} / \mathrm{cm}^{3}$, a factor of ten over the conventional power density. The result is a fabricated equipment which has the dimensions of $7.8 \times 6 \times 10.7 \mathrm{~cm}\left(500 \mathrm{~cm}^{3}\right)$, and achieved an efficiency of $95 \%$ for an output of $4.3 \mathrm{~kW}$. Consequently, the power density was $8.6 \mathrm{~W} / \mathrm{cm}^{3}$, and is close to the target. The further improvement is expected in the future.

(2) A new DC circuit breaker was developed by using SiC-SIT device. The surge-voltage reduction technique by varying the gate-voltage during the punch-through mode was developed, and the DC circuit breaker without a snubber circuit has been realized. Replacing the conventional mechanical circuit breaker and the conventional semiconductor one with the new SiC-SIT circuit breaker introduces additional functions such as the high intelligence via a network. The next-generation DC power system with "Power Routing Control" is expected to be developed on the basis of new ideas mentioned above.

\section{REFERENCES}

[1] The Green Initiative, Ministry of Economy, Trade and Industry, December 2007.

[2] N.Yamato, et al.: "Effect of Breaking High Voltage Direct Current (HVDC) Circuit on Demonstrative Project on Power Supply Systems by Service Level in Sendai", IEEE 29th International Telecomunication Conference 2007, pp. 46-51

[3] A.Fukui et al.: "Smaller DC Semiconductor Circuit Breakers," 2009 IEICE General Conference, Collected Presentations 2, pp. 317 Conference 2007, pp. 46-51

[4] Y.Tanaka, et al.: "700-V 1.0-m $\Omega \cdot \mathrm{cm} 2$ Buried Gate SiC-SIT (SiC-BGSIT)", IEEE Electron Device Letters, VOL. 27, NO. 11, pp. $908-910$

[5] A.Matsumoto, A.Fukui, T.Takeda, M.Yamasaki: "Development of 400-Vdc Output Rectifier for $400-\mathrm{Vdc}$ Power Distribution System in Telecom Sites and Data Centers," Proc. INTELEC'10, No.4-3, June.2010.

[6] R.Simanjorang, H.Yamaguchi, H.Ohashi, N.Nakao, T.Ninomiya, S.Abe, M.Kaga, A.Fukui: "High-efficiency high-power dc-dc converter for energy and space saving of power-supply system in a data center,'APEC'11 Record, pp.600-605, Mar. 2011

[7] Y.Sato, S.Tobayashi, Y.Tanaka, A.Fukui, M.Yamasaki, H.Ohashi: "An Investigation of SiC-SIT DC Circuit Breakers for Higher Voltage Direct Current Distribution Systems," ECCE'10 Record, pp.3290-3295, Sept.2010. 